Research, Society and Development, v. 9, n.1, e02911504, 2020

(CC BY 4.0) | ISSN 2525-3409 | DOI: http://dx.doi.org/10.33448/rsd-v9i1.1504

\title{
Efeito fitoterápico de plantas medicinais sobre a ansiedade: uma breve revisão
} Effect of herbal medical plants on anxiety: a brief review

Efecto de las plantas medicinales a base de hierbas sobre la ansiedad: una breve revision

Recebido: 13/08/2019 | Revisado: 29/08/2019 | Aceito: 01/09/2019 | Publicado: 20/09/2019

\section{Mariana Matos Bortoluzzi}

ORCID: https://orcid.org/0000-0002-8722-3658

Centro Universitário Campo Real. Guarapuava, Paraná, Brasil.

E-mail: maarianabortoluzzi.mats@hotmail.com

Vania Schmitt

ORCID: https://orcid.org/0000-0003-3388-9558

Centro Universitário Campo Real. Guarapuava, Paraná, Brasil.

E-mail: vania_schmitt@hotmail.com

Caryna Eurich Mazur

ORCID: https://orcid.org/0000-0002-1278-5963

Universidade Estadual do Centro-Oeste (UNICENTRO). Guarapuava, Paraná, Brasil.

E-mail: carynanutricionista@gmail.com

\section{Resumo}

A ansiedade é um dos sintomas mais comuns associados ao estilo de vida moderno. Em contrapartida, percebe-se o aumento pela procura de práticas integrativas e complementares como a fitoterapia para amenizar os sintomas associados à ansiedade. Dessa maneira, esse artigo tem por objetivo listar os fitoterápicos mais utilizados no tratamento da ansiedade. Trata-se de uma revisão de literatura dos últimos 18 anos, onde foi pesquisado sobre o funcionamento e a aplicação de fitoterápicos e seus efeitos benéficos para promoção de saúde, especialmente na ansiedade. Entre os fitoterápicos de efeito carminativos estão a valeriana (Valeriana officinalis), hortelã (Mentha) e camomila (Matricaria chamomilla), Flor de Laranjeira (Citrus $X$ sinensis), Erva Cidreira (Melissa officinalis) e Capim Limão (Cymbopogoncitratus) que podem tratar de dores agudas e serem sedativas, ainda, o maracujá (Passiflora edulis), e em casos de insônia inclui-se também espinheiro-branco (Crataeguslaevigata), e lúpulo (Humuluslupulus). Em relação aos compostos bioativos, a utilização de alimentos fermentados, de óleos essenciais - especialmente ômega 3, flavonoides, mostraram-se potenciais tratamentos alterativos para a ansiedade. 
Palavras-chave: Depressão; Fitoterapia; Sistema nervoso.

\begin{abstract}
Anxiety is one of the most common symptoms associated with the modern lifestyle. In contrast, there is an increase in the search for integrative and complementary practices such as herbal medicine to alleviate the symptoms associated with anxiety. Thus, this article aims to list the most used herbal medicines in the treatment of anxiety. This is a literature review of the last 18 years, where it was researched about the functioning and application of herbal medicines and their beneficial effects for health promotion, especially in anxiety. Carminative herbal remedies include valerian (Valeriana officinalis), mint (Mentha) and chamomile (Matricaria chamomilla), orange blossom (Citrus X sinensis), lemon balm (Melissa officinalis) and lemongrass (Cymbopogoncitratus). acute pains and sedatives, including passion fruit (Passiflora edulis), and in cases of insomnia include also white hawthorn (Crataeguslaevigata), and hops (Humuluslupulus). In relation to bioactive compounds, the use of fermented foods, essential oils - especially omega 3 flavonoids, have been shown to be potential alternative treatments for anxiety.
\end{abstract}

Keywords: Depression; Phytotherapy; Nervous system.

\title{
Resumen
}

La ansiedad es uno de los síntomas más comunes asociados con el estilo de vida moderno. En contraste, hay un aumento en la búsqueda de prácticas integradoras y complementarias como la medicina herbal para aliviar los síntomas asociados con la ansiedad. Por lo tanto, este artículo tiene como objetivo enumerar las hierbas medicinales más utilizadas en el tratamiento de la ansiedad. Esta es una revisión de la literatura de los últimos 18 años, donde se investigó sobre el funcionamiento y la aplicación de las hierbas medicinales y sus efectos beneficiosos para la promoción de la salud, especialmente en la ansiedad. Los remedios herbales carminativos incluyen valeriana (Valeriana officinalis), menta (Mentha) y manzanilla (Matricaria chamomilla), azahar (Citrus X sinensis), bálsamo de limón (Melissa officinalis) y hierba de limón (Cymbopogoncitratus). dolores agudos y sedantes, también fruta de la pasión (Passiflora edulis), y en casos de insomnio también incluyen espino blanco (Crataeguslaevigata) y lúpulo (Humuluslupulus). En relación con los compuestos bioactivos, se ha demostrado que el uso de alimentos fermentados, aceites esenciales, especialmente flavonoides omega 3, son posibles tratamientos alternativos para la ansiedad.

Palabras clave: depresión; Medicina herbaria; Sistema nervioso. 


\section{Introdução}

Os conhecimentos sobre as plantas medicinais, assim como o seu uso em forma de medicamento, têm acompanhado a humanidade ao longo dos anos. Entretanto, recentemente vem ocorrendo um retorno a essa utilização por grande parte da população, ganhando espaço no mercado que havia sido dominado por produtos industrializados (Carneiro et al., 2014). Trata-se de recursos medicinais alternativos para o tratamento de diversas enfermidades e, muitas vezes, em algumas comunidades passam a ser a solução mais acessível em relação aos medicamentos alopáticos. Muitas espécies vegetais possuem propriedades terapêuticas, e, portanto, o uso de plantas medicinais representa um fator essencial para a manutenção das condições de saúde das pessoas (Morelli, 2010).

A planta medicinal segundo a Agência Nacional de Vigilância Sanitária (ANVISA) é toda planta ou partes da mesma que contenham as substâncias responsáveis pela ação terapêutica (Brasil, 2017). De acordo com a Organização Mundial de Saúde, 80\% da população mundial faz uso de algum tipo de planta medicinal. O uso dessas ervas é feito na maioria das vezes por adultos e idosos que buscam complementar o tratamento de uma doença crônica (Rodrigues, 2000; Carneiro et al., 2014).

$\mathrm{O}$ efeito de uma planta medicinal é determinado pela forma a qual a espécie é usada, desde sua posologia e preparo (Lemnis, 2016). Assim, para se ter uma garantia no uso desses fitoterápicos e remédios derivados deles é necessário não apenas medidas de controle, mas a realização de campanhas que visem informar a população de seus riscos e benefícios, e os profissionais de saúde devem ter educação contínua sobre esses elementos. Estudos com plantas medicinais alternativas tem merecido cada vez mais atenção, devido às informações que fornecem a ciência (Carneiro et al., 2014).

Fisiologicamente, a diminuição da serotonina e o aumento do cortisol constituem gatilhos para o aumento da ansiedade, depressão e estresse. Sabe-se que a alimentação é capaz de diminuir os quadros de ansiedade. O excesso de açúcar, gordura saturada, trans e ácidos como os contidos em refrigerantes podem piorar o quadro. Em contrapartida, uma alimentação rica em fibras, peixes de águas profundas - ricos em ômega 3, frutas e hortaliças, podem promover melhora da sintomatologia (Rodrigues, 2000; Morelli, 2010; Lemnis, 2016).

Com isso, o presente estudo tem por objetivo fornecer informações sobre as tendências dos estudos com plantas medicinais e óleos essenciais que favoreçam no controle da ansiedade. 


\section{Metodologia}

Trata-se de um artigo de revisão bibliográfica simples, onde foi pesquisado sobre o funcionamento e a aplicação de fitoterápicos e seus efeitos benéficos para promoção de saúde, especialmente na ansiedade. Como suporte para esse artigo foi percorrida metodologia descrita por Pereira et al. (2018).

Foram analisados livros-textos, documentos governamentais, artigos científicos em bases de pesquisas como o Google Acadêmico, Scielo e Lilacs.

Foram incluídos artigos e textos publicados entre os anos 2000 a 2018. Também foram incluídas na pesquisa cartilhas, teses, dissertações, informações ou documentos de sites governamentais. No início os termos utilizados em português foram: "fitoterapia e ansiedade", "ansiedade e plantas medicinais", "estresse e fitoterapia". A partir disso, foram selecionados outros termos: "Passiflora edulis", "Citrus Aurantiun L", "Mentha", "Melissa Officinalis L", "Matricaria chamomilla", "Cymbopogon citratus" e "óleos essenciais e ansiedade". Foram excluídos artigos indisponíveis na íntegra e artigos de políticas. Os resultados foram expressos de maneira descritiva.

\section{Resultados e Discussões}

\subsection{Fitoterapia}

A fitoterapia é uma forma de tratamento simples e natural, onde busca-se tratar ou prevenir doenças a partir da preparação de vegetais ou aos princípios ativos que deles possam ser extraídos (Brandão, 2015).

O fitoterápico é o medicamento obtido exclusivamente de derivados de drogas vegetais. É caracterizado pelo conhecimento da eficácia e dos riscos de seu uso, como a reprodução da sua qualidade. Não se pode considerar um fitoterápico aquele que inclui substâncias isoladas de qualquer origem, ou associações com extratos vegetais ou encapsulados. O fitoterápico está associado ao efeito terapêutico, que pode ser utilizado como controle de qualidade de suas matérias primas. Tem como marcador, o princípio ativo, as substâncias, ou compostos quimicamente caracterizados com farmacologia conhecida, principalmente pelos efeitos terapêuticos e colaterais. Na maioria das vezes, a ação é devida a um conjunto de moléculas (fitocomplexo) que agem sinergicamente para promover a ação 
Research, Society and Development, v. 9, n.1, e02911504, 2020

(CC BY 4.0) | ISSN 2525-3409 | DOI: http://dx.doi.org/10.33448/rsd-v9i1.1504

terapêutica e, às vezes, antagonicamente, neutralizando determinados efeitos tóxicos (Rodrigues, 2000).

Assim, a busca constante por plantas medicinais ou fitoterápicos pode ser considerada uma prática integrativa e complementar de cuidado, especialmente nas doenças crônicas. Entretanto, a utilização da fitoterapia é sempre adicional ao tratamento clínico convencional, nesse sentido, as plantas medicinais podem tratar os sintomas e não trazer a cura.

\subsection{Plantas com Efeito Sedativo}

Pode-se encontrar na composição das plantas medicinais vários elementos que agem de diferentes formas nas zonas do sistema nervoso. Pode-se citar as que tem efeito sobre os nervos motores do sistema muscular, nos casos de sensação de dor e espasmos digestivos (Zeraik et al., 2010).

Entre os fitoterápicos de efeito carminativos estão a valeriana (Valeriana officinalis), hortelã (Mentha) e camomila (Matricaria chamomilla), Flor de Laranjeira (Citrus X sinensis), Erva Cidreira (Melissa officinalis) e Capim Limão (Cymbopogoncitratus) que podem tratar de dores agudas e serem sedativas, ainda, o maracujá (Passiflora edulis), e em casos de insônia inclui-se também espinheiro-branco (Crataeguslaevigata), e lúpulo (Humuluslupulus) (Zeraik et al., 2010).

Considerando a intensa utilização das plantas medicinais no Brasil para a ansiedade e a sua relevância para manutenção da saúde de várias comunidades, busca-se abordar nesse artigo as suas propriedades que beneficiam e previnem tais desequilíbrios orgânicos e/ou doenças, bem como, atividades empíricas de prepará-las de uma forma simples e prática, para assim utilizar os elementos fundamentais para a promoção da saúde humana.

\subsection{Ansiedade}

A ansiedade é uma das mais frequentes desordens psiquiátricas cuja etiologia depende de uma influência mútua entre predisposição individual e fatores ambientais. No Brasil, os transtornos ansiosos encontram-se entre os mais prevalentes diagnósticos psiquiátricos, sendo as mulheres mais afetadas que os homens (Brandão, 2015).

A ansiedade é considerada normal quando é manifestada por meio de respostas do organismo, e é considerada patológica quando a intensidade ou a frequência não corresponde aos motivos que a desencadeou, ou quando não há motivos aparentes. Quando ela traz 
sofrimento, desentendimento, prejuízo ao comportamento, fuga de situações importantes é caracterizada como doença (Bulasmed, 2016).

Por isso, buscar alternativas complementares para o tratamento da ansiedade é uma necessidade clínica. A utilização de fitoterápicos podem ser auxiliar para o tratamento dos sintomas que envolvam a ansiedade, principalmente o estresse, a taquicardia, o nervosismo e o mal-estar psicológico geral.

\subsection{Passiflora edulis (Maracujá)}

A Passiflora edulis ou mais popularmente conhecida como maracujá ou também como flor da paixão é muito utilizada como calmante, os extratos de suas folhas estão sendo focos da pesquisa. O maracujá é um fruto de grande interesse econômico e social, tem compostos como polifenois, ácidos graxos poli-insaturados e fibras que possuem funcionalidade terapêutica (Silva, 2015).

A família Passifloraceae distribui-se, principalmente, por regiões tropicais nas Américas, África e Ásia. Esta família possui cerca de 600 espécies e 20 gêneros (Masson, 1998).

Seu cultivo é realizado pelo plantio de sementes em local ensolarado e fértil, preferencialmente em lugares quentes. Seu florescimento ocorre na primavera ao final do outono com a frutificação (Boorhen, 1999).

Esta planta contém compostos bioativos tais como a passiflorina que é uma substância semelhante à morfina, além de possuir alcaloides, glucosídeos, flavanóides, alpha-alanina, apigenina, arabidina, ácido cítrico, cumarina, glutaminamharmanina, ácidos fenólicos, pectina e outros. O maracujá também possui seratonina e maracujina, o que pode promover efeito calmante, mas sem dependência, os alcaloides podem causar a diminuição da pressão arterial, ou seja, possuem propriedades antiespasmódicas. As folhas são de uso medicinal, e podem ser usadas como calmante em forma de chás (Boorhen, 1999). É indicada para ansiedade, insônia, hipertensão arterial, taquicardia, palpitações, mialgias.

Esse fruto detém propriedades depressoras do Sistema Nervoso Central que atua na parte dos distúrbios da ansiedade, na sedação e na convulsão, ou seja, tem propriedades ansiolíticas e sedativas (Panizza, 1998), o que resulta em uma ação tranquilizante e antiespasmódica da musculatura lisa (Lorenzi et al., 2006).

O mecanismo de ação da passiflora é a inibição da monoamina oxidase e a ativação dos receptores de GABA (Ácido gama-aminobutírico) este é o principal neurotransmissor 
inibitório, agindo na interrupção de circuitos neurais, assim com os baixos níveis do GABA se associam com a ansiedade (Matos, 2002).

Para o uso da passiflora é indicado $3 \mathrm{~g}$ de folhas secas para $150 \mathrm{ml}$ de água, isso para o uso da passiflora alata, p. edulis. Para a passiflora incarnata, que é a mais comum é indicado de 6 a 9g da parte seca em $150 \mathrm{ml}$ de água. O método para elaboração do chá é por infusão. Quando em infusão tomar de 50 a 200ml/dia e quando tintura tomar de 2 a 10ml/dia (Barbosa Filho; Borba, 1992).

Pacientes com histórico de hipersensibilidade e alergia, gestantes, ou lactantes, não deverão fazer uso sem orientação médica, não deve ser utilizado junto a bebidas alcoólicas. Também não deve ser associado a outros medicamentos com efeito sedativo, hipnótico e antihistamínico. Crianças menores de 12 anos não devem usar sem orientação médica (Brasil, 2017).

Por se tratar de uma fruta amplamente conhecida em todo mundo, a utilização de outras partes da planta (caule, folhas e sementes) também pode ser coadjuvante no tratamento da ansiedade. A utilização em excesso pode causar sonolência.

\subsection{Citrus Aurantiun L. (flor de laranjeira)}

Seu nome científico é Citrus Aurantiun L., mas também pode ser encontrada por laranja-amarga, laranja-da-terra, laranja-azeda, laranja-bigarade e laranja-de-sevilha (Masson et al., 1998).

Da laranjeira pode-se aproveitar quase que todas as partes, desde o fruto, até as folhas, e é na medicina tradicional chinesa que é utilizada como folha aromática, amarga, digestiva, expectorante, diurética e hipotensiva, sendo utilizada também como calmante (Brasil, 2017).

Para a infusão é sugerido extrair as folhas, e tem como propriedades sudorífica, antigripal, carminativa e antiespasmódica (Ceolin et al., 2009). Do extrato dos botões, passado pelo processo de maceração em água por média de 3 a 4 horas, é indicado na prática de ação calmante, em casos de insônia e nervosismo (Gadelha, 2015).

A flor de laranjeira, dessa maneira, pode ser uma possibilidade para o tratamento da ansidade por ter ação tranquilizante leve.

\subsection{Mentha (hortelã)}

Conhecido pelo seu nome científico de mentha $x$ villosaHuds, e no popular como hortelã-rasteira, hortelã-de-panela ou menta-vilosa (Masson et al., 1998). 
Research, Society and Development, v. 9, n.1, e02911504, 2020

(CC BY 4.0) | ISSN 2525-3409 | DOI: http://dx.doi.org/10.33448/rsd-v9i1.1504

Tem grande importância na ação contra microrganismos, especialmente, os intestinais (Lins, 2015). Apresenta efeito em ações espamolíticas, antivomitiva, carminativa, estomáquica e anti-helmíntica (Carneiro, 2015). Assim, a utilização do hortelã pode ser parte do tratamento da ansiedade, especialmente por sua ação calmante. Como a ansiedade também pode provocar distúrbios no sistema gastrintestinal, o hortelã também pode auxiliar por sua ação digestiva.

\subsection{Melissa Officinalis L. (erva cidreira)}

Entre todas as espécies que são consideradas medicinais, a erva cidreira (Melissa Officinalis L.) se destaca. É uma planta herbácea perene, aromática, ramificada desde a base, ereta ou com ramos ascendentes, de $30 \mathrm{~cm}$ a $60 \mathrm{~cm}$ de altura. Folhas membranáceas rugosas de $3 \mathrm{~cm}$ a $6 \mathrm{~cm}$ de comprimento, de cor verde escuras na parte superior e verde claro na inferior (Costa, 2007).

As suas folhas inflorescências são empregadas na forma de chá, de preferência com a planta fresca, como calmante nos casos de ansiedade e insônia, e como medicação contra dispepsia, gripe, bronquite crônica, cefaleias, enxaqueca, dores reumáticas, para normalizar as funções gastrointestinais e no tratamento de manifestações virais (Costa, 2007).

A infusão é com uma colher se sobremesa de erva cidreira, sendo as folhas e ramos frescos ou secos bem picados. Recomenda-se também como banho relaxante de imersão durante 15 minutos, preparado pela adição de meio litro de água fervente sobre 15 colheres de sopa de erva cidreira com falhas, ramos e flores picados. Seus taninos diferenciados atribuem fortes ações virustáticas. Na sua composição química é encontrada a presença de óleo essencial rico e citral, citronelal, citronelol, limoneno, linanol e geraniol, taninos, ácidos triterprenoides, flavonoides, mucilagens, resinas e substâncias amargas, bem como glicosídeos dos álcoois presentes nos óleos essências (Bown, 1995).

A melissa torna-se, assim, um dos fitoterápicos mais utilizados no tratamento da ansiedade, especialmente por sua palatabilidade e alta aceitação sensorial. Ainda, pode melhorar as crises de dores de cabeças e também insônia, sintomas comum em indivíduos que sofrem de ansiedade.

\subsection{Matricaria chamomilla (camomila)}


A camomila (Matricaria chamomilla) teve seus primeiros fins descobertos pelos egípcios, gregos e romanos, e era utilizada como aromatizante, em cosméticos e medicamentos. Além disso, é usada em licores, dentifrícios para a higiene bucal por ser conhecida como planta curativa e calmante (Arnous; Santos \& Beinner, 2005).

Para fins curativos a planta é recomendada para quem tem asma, doenças neurológicas, náuseas, doenças dermatológicas, entre outros. A planta tem ação antiinflamatória, antialérgica, antibacteriana, sedativa e atua também como relaxante muscular. É recomendada para melhorar a digestão, aliviando dores ou náuseas e vômitos, além de aliviar cólicas menstruais, gastrites, úlceras gástricas, entre outros (Morais, 2006).

O chá de camomila vem sendo estudado para ajudar quem sofre de ansiedade e insônia. Essas causas são relacionadas com o dia a dia, estresse, cansaço, estilo de vida e funções vitais do corpo (Lemnis, 2016). Logo, a camomila é uma importante aliada nos sintomas relacionados à ansiedade, especialmente por suas funções carminativas.

\subsection{Cymbopogon citratus (capim limão)}

A espécie Cymbopogon citratus é nativa das regiões tropicais da Ásia, no Brasil é conhecida como Capim Limão, capim-cidró, capim cidreira, erva cidreira, capim cheiroso, apresentando grande potencial, pois é utilizada na medicina popular como calmante, sedativa e ansiolítica (Brandão, 2015).

É utilizado como calmante, analgésico (em dores gástricas, abdominais e cefaleia), antifebril, carminativo, digestivo, hipotensor, bactericida em conjuntivites. Também possui efeito antiespasmódico, combate o histerismo e outras afecções nervosas (Brandão, 2015).

\section{9 Óleos essenciais}

Estudos concluem que os óleos essenciais podem proteger a saúde a longo prazo, assim como suavizar sintomas de males comuns. Eles ajudam a aliviar dores, melhorar o humor e diminuir náuseas. Aromas diferentes estimulam respostas distintas no cérebro. Alguns estudos ainda relatam o efeito dos óleos no estresse, aumento de imunidade, E. Coli e células cancerígenas. Trabalhos mostram que, quando inalados, os óleos ativam o hipotálamo, que controla os hormônios, a energia e a motivação (Agatonovic-Kustrin; Kustrin; Morton, 2019). 
A família da menta é uma família de plantas aromáticas que contém a maior parte das ervas culinárias encontradas na dieta mediterrânea. Sendo considerada uma das as dietas mais saudáveis do mundo, e é encontrado para ser benéfico não só para o coração, mas também para o cérebro. Estas ervas são ricas em antioxidantes que podem prevenir o dano oxidativo causado pelos radicais livres. No entanto, eles também contêm compostos biologicamente ativos com potente $\alpha$-amilase e atividades inibitórias da acetilcolinesterase (AgatonovicKustrin; Kustrin; Morton, 2019).

O consumo de ervas frescas pode ajudar a melhorar a memória e reduzir níveis de açúcar no corpo. O uso de ervas como alimento funcional pode levar a melhorias significativas saúde. A estimulação cognitiva com alimentos médicos e ervas medicinais pode atrasar o desenvolvimento de declínio, e melhorar a qualidade de vida dos pacientes com doença de Alzheimer. Os óleos essenciais têm sido utilizados por gerações para facilitar os sintomas de DA (doença de Alzheimer), e inibem a alfa-amilase em grau significativo. Inibição de enzimas digestivas do amido, como $\alpha$-amilase, pode desempenhar um papel importante no controle do diabetes (Braga, 2010).

Os alimentos fermentados à base de plantas, como vegetais fermentados e frutas, desempenham um papel significativo na nutrição humana, fornecendo vitaminas, minerais, oligoelementos e outros nutrientes essenciais. A fermentação de alimentos à base de plantas é uma maneira geral de preservar e expandir as características sensoriais dos alimentos, além disso possuem propriedades para diminuição do estresse e ansiedade (Agatonovic-Kustrin; Kustrin; Morton, 2019).

Consequentemente, a utilização de óleos essenciais na ansiedade deve ser analisada com ponderação e sempre informando ao médico, pois podem haver alterações com os medicamentos tradicionais. Tanto seus potenciais efeitos positvos quanto negativos devem ser levados em consideração para a integralidade do cuidado e garantia de melhora dos sintomas.

\section{Considerações Finais}

Os efeitos dos fitoterápicos na promoção e manutenção da saúde, especialmente na ansiedade, vem sendo essenciais para tratamentos terapêuticos complementares. $\mathrm{O}$ consumo de ervas medicinais frescas pode ajudar a melhorar a ansiedade, este efeito é melhorado se combinado com o uso de óleos essenciais. Esta revisão destaca o uso de fitoterápicos como tratamento alternativo da ansiedade. 
Research, Society and Development, v. 9, n.1, e02911504, 2020

(CC BY 4.0) | ISSN 2525-3409 | DOI: http://dx.doi.org/10.33448/rsd-v9i1.1504

Dessa maneira, conclui-se que existem vários fitoterápicos que auxiliam no quadro de ansiedade. Entretanto, é preciso que haja a condução de mais estudos para a comprovação da eficácia terapêutica, especialmente em seres humanos.

\section{Referências}

Agatonovic-Kustrin, S., Kustrin, E., Morton, D.W. (2019). Essential oils and functional herbs for healthy aging. Neural Regeneration Research, 14 (3), 441-445.

Arnous, A.H; Santos, A.S; Beinner, R.P.C. (2005). Plantas Medicinais de uso caseiroconhecimento popular e interesse por cultivo comunitário. Revista Espaço da Saúde, 6 (2), 16.

Barbosa Filho, J.M., Borba, M. (1992). Constituintes Químicos de Extrato de Butanólico de Mentha X Vilosa Huds. Curitiba.

Boorhen, R.L. (1999). Segredos e Virtudes das Plantas medicinais. Rio de Janeiro, RJ, Reader's Digest Brasil Ltda.

Bown, D. (1990). The Herbs Society of America- Encyclopedia of Herbs \& Theis Uses. Dorling Kindersley Publishing Inc; New York.

Braga, J.F.E., Pordeus, L.C., Silva, A.T.M., Pimenta, F.C.F., Diniz, M.F.F.M., Almeida, R.N. (2010).Ansiedade Patológica: bases neurais e avanços na abordagem psicofarmacológica. Revista Brasileira de Ciências da Saúde, 14 (2), 93-100.

Brandão, L.E.M. (2015). Avaliação dos efeitos do extrato de Passiflora cincinnata Masters em camundongos: efeitos na ansiedade e potencial neuroprodutor. [Dissertação de mestrado] PósGraduação em Psicobiologia. Universidade Federal do Rio Grande do Norte. Natal.

Brasil. (2017). Agência Nacional de Vigilância Sanitária (ANVISA). Lista DCB Plantas Medicinais atualizada em dezembro 2017. Brasília. Disponível em: http://portal.anvisa.gov.br/resultado-de-

busca?p_p_id=101\&p_p_lifecycle $=0 \& p \_p \_s t a t e=$ maximized $\&$ p_p_mode $=$ view $\&$ p_p_col_id $=$ column-

1\&p_p_col_count=1\&_101_struts_action=\%2Fasset_publisher\%2Fview_content $\&$ 101_asset EntryId=3881905\&_101_type=document Acesso em 13.junho.2019.

Bulasmed. (2018). BulasMed Referências completas de medicamento. [Online]. Available: http://www.bulas.med.br/.Acesso em 08 Junho 2018. 
Carneiro, F. M., Silva, M. J. P., Borges, L., Lalbernaz, L. C., Costa, J. A. (2014). Tendências dos Estudos com Plantas Medicinais no Brasil. Revista Sapiência: sociedades, saberes e práticas educacionais, 3 (2), 44-75.

Ceolin, T., Heck, R.M., Barbieri, R.L., Souza, A.D.Z., Rodrigues, W.F., Vanini, M. (2009). Plantas Medicinais utilizadas como calmantes por agricultores ecológicos da região Sul do Rio Grande do Sul. Journal of Nursing UFPE, 3(4), 1034-4.

Costa, C.A.R.A. (2019). Estudo da ação ansiolítica e sedativa de preparações obtidas de Cymbopogon Citratus (D. C.). [Dissertação de mestrado] Instituto de Biociências Departamento de Farmacologia - Universidade Estadual de São Paulo. Acesso em 08 Junho 2019.

Gadelha, C. S., Pinto Junior, V.M., Bezerra, K.K.S., Maracajá, P.B., Martins, D.S.S. (2015). Utilização de Medicamentos Fitoterápicos e Plantas em Diferentes Segmentos da Sociedade. Revista Verde de Agroecologia e Desenvolvimento Sustentável, 10 (3), 01 - 15.

Lemnis (2016). "LEMNIS Farmácia". [Online]. Disponível: http://www.lemnisfarmacia.com.br/passiflora-maracuja-\%E2\%80\%93-no-tratamento-dainsonia-ansiedade-irritabilidade-e-insonia/. Acesso em 08 Junho 2016.

Lins, A.D.F., Oliveira, M.N., Fernandes, V.O., Rocha, A.P.T., Sousa, F.C., Martins, A.N.A., Nunes, E.R. (2015). Quantificação de Compostos Bioativos em Erva Cidreira (Melissa offcinalis L.) e Capim Cidreira (Cymbopogon citratus Stapf). Revista da UFPB - Gaia Scientia, 17-21.

Masson, A.S., Garcia, A., Vana, C., Salazar, J.G.I.S., Cobo, R.M., Martínez, C.A., Garcia, J.E. (1998). Valdemecum de prescriocíon plantas medicinales. Barcelona.

Matos, F.J.A. (2002). Plantas Medicinais: Guia de Seleção de Emprego de Plantas Usadas em Fitoterapia no Nordeste do Brasil. Imprensa Universitária/ Edições UFC.

Maury, E. A, Rudder, C. (2002). Guia de plantas medicinais. Editora Riddel: São Paulo.

Morais, T.C; Vieira, M.C; Heredia, Z; Teixeira, I.R; Ramos, R.B.M. (2006). Produção de Biomassa e teor de óleos essenciais da camomila (Chamomilla Recutia L. Rauschert) em função das adubações com fósforo e nitrogênio. Revista Brasileira de Plantas Medicinais, 8 (4), 120-125. 
Morelli, M.S. (2010). Guia de produção para plantas medicinais, aromáticas e flores comestíveis. Porto Alegre, RS: Cidadela.

Panizza, S. (1998). Plantas que curam (Cheiro de Mato). São Paulo: Ibrasa.

Pereira, A.S. et al. (2018). Metodologia do trabalho científico. [e-book]. Disponível em: https://repositorio.ufsm.br/bitstream/handle/1/15824/Lic_Computacao_MetodologiaPesquisa-Cientifica.pdf? sequence=1. Acesso em: 29 ago. 2019.

Rodrigues, C. A. (2000). A Cultura do Maracujazeiro. Empresa de Pesquisa Agropecuária de Minas Gerais. Belo Horizonte.

Silva, J.D. (2015). Efeitos da Suplementação da Passiflora Incarnata L. sobre a ansiedade em Humano. [dissertação de mestrado] Programa de Pós-Graduação em Ciências da Nutrição. Universidade Federal da Paraíba. João Pessoa.

Sivamaruthi, B.S., Kesika, K., Chaiyasut, C. (2018). Impact of Fermented Foods on Human Cognitive Function: A Review of Outcome of Clinical Trials. Scientia Pharmaceutica, 86 (3).

Souza, V.C; Lorenzi, H. (2008). Botânica sistemática: Guia ilustrado para identificação das famílias de Fanerógamas nativas e exóticas no Brasil. Baseado em APG II. 2nd ed. Instituto Plantarum, Nova Odessa.

Zeraik, M.L, Pereira, C.A.M., Zuin, V.G., Yariwake, J.H. (2010). Maracujá: um alimento funcional? Revista Brasileira de Farmacologia, 20 (3), 459-71.

\title{
Porcentagem de contribuição de cada autor no manuscrito
}

\author{
Mariana Matos Bortoluzzi - 50\% \\ Vania Schmitt $-15 \%$ \\ Caryna Eurich Mazur - 35\%
}

